\title{
Studying the effects of the lung mass on the absorbed dose to the lung due to the administration of ${ }^{131}$ I for therapeutic purposes
}

\author{
Z. Sajjadi ${ }^{1 \star}$, H. Miri-Hakimabad ${ }^{2}$ and L. Rafat-Motavalli ${ }^{2}$ \\ 1 Department of Physics, Payame Noor University (PNU), 19395-3697, Tehran, Iran. \\ 2 Department of Physics, School of Sciences, Ferdowsi University of Mashhad, 91775-1436 Mashhad, Iran.
}

Received 5 November 2015 - Accepted 17 June 2016

\begin{abstract}
I is a radiopharmaceutical used for the treatment of advanced lung cancer, resulting in high organ doses. The effects of the lung mass on the absorbed dose to the lung due to the administration of ${ }^{131}$ I were studied in this research. For this purpose, the lung was selected as the source of ${ }^{131}$ I. Furthermore, 98 similar mathematical phantoms, only different in their lung mass, were developed. The received dose per decay of ${ }^{131} \mathrm{I}$ for each organ was calculated using MCNPX. The results indicate that for the electrons emitted from the decay of ${ }^{131} \mathrm{I}$, the dose changes proportionally to the inverse of the lung mass. Considering that the participation of the electrons resulting from the decay of ${ }^{131} \mathrm{I}$ in the amount of the lung dose outweighs the photon participation by a great deal, changes in the dose for the sum of the electrons and photons per decay are proportional to the inverse of the lung mass, as for the electrons.
\end{abstract}

Keywords: Phantom / internal dosimetry / Monte Carlo / absorbed dose

\section{Introduction}

Lung cancer is one of the most common types of cancer, which causes a quarter of cancer-related deaths (Siegel et al., 2014). The number of those who are suffering from this cancer is increasing day by day due to the growth of smoking and industrial pollution. Especially among men, lung cancer is known to be the most common type of fatal cancer (Halperin et al., 2013). Several methods are applied for the diagnosis and treatment of lung cancer, in most of which the lung is irradiated.

On the other hand, the high vulnerability of the lung to radiation increases the risk of developing a fatal secondary cancer caused by radiation (Siegel et al., 2014). The possibility of fatal secondary malignancies related to radiation therapy is estimated to be $0.85 \%$ per Sievert (Kry et al., 2005). Hence, accurate calculations of dosimetry and determining the effective parameters of the dose are of vital importance for evaluating the risk of using radiopharmaceuticals for lung cancer treatment (Han, 2005).

Nowadays, internal dosimetry calculations are made using reference computational phantoms of adults, children and pregnant women, which are developed based on the data of the 50th percentile of a population (ICRP, 1975, 2002). Tomography images and the samples acquired from human corpses are indicative of the fact that the size, shape and positions of human organs are significantly different from one person to another. These deviations have not been considered in

\footnotetext{
^h_sajadi@pnu.ac.ir
}

the current models (Mofrad et al., 2010; Ebrahimi-Khankook et al., 2015). It is very important to investigate to what degree this anatomical difference can affect the deviation of the absorbed dose.

It is worth noting that there are other factors that affect the amount of the organ dose such as the biodistribution of a radiopharmaceutical, and the size and shape of the organs. Nevertheless, one of the most prominent parameters affecting the organ dose is the mass of the organ, which is more remarkable in comparison with biokinetic parameters (Brill et al., 2006; Stabin, 2008).

There are many reference phantoms, each of which has been developed based on the anatomical data of patients from a specific region of the world. All reference phantoms of a particular race (Caucasian or Asian) have similar wholebody sizes, but these phantoms can be remarkably different in the received dose since their anatomical structures were based on different patients. Research has also confirmed that in many cases, the dose received by a patient differed from the calculated dose based on the appropriate reference phantom (Ebrahimi-Khankook et al., 2014).

There are scaling laws which allow adjusting the $S$ values of reference models to patients. These are implemented in software such as OLINDA/EXM. Although it is possible to do mass correction for the dose calculation, in most clinical centers a similar dose is administered to different patients (Chen et al., 2005, Yu et al., 2006).

In this project, the degree of changes in the lung dose in relation to its mass changes was studied for the lung as the 
source of ${ }^{131} \mathrm{I}$. Since iodine is mainly absorbed by the thyroid, in order for the lung to be considered as the source of ${ }^{131} \mathrm{I}$, the patients receive potassium to increase the lung intake of iodine (Chen et al., 2005; Yu et al., 2006).

\section{Materials and methods}

${ }^{131}$ I-labeled tumor necrosis therapy chimeric antibody $\left({ }^{131} \mathrm{I}\right.$-chTNT) is a radiopharmaceutical used for the treatment of advanced lung cancer. ${ }^{131} \mathrm{I}$ is a $\beta$-emitting radionuclide, the electrons of which can be used for tumor necrosis and its longrange gamma rays can be used for imaging. Since, in general, the majority of the iodine is absorbed by the thyroid, to prevent the thyroid from absorbing the iodine, the patient receives potassium two days before and after the injection of ${ }^{131}$ I-chTNT (Chen et al., 2005; Yu et al., 2006). Hence, the absorption rate of the iodine will increase in the lung. In this way, the lung is considered as the source of ${ }^{131} \mathrm{I}$.

In this study, the adult ORNL phantom which was developed by Cristy and Eckerman (Loevinger et al., 1956; Cristy, 1985; Stabin et al., 1995) and updated by Miri-Hakimabad and Rafta-Motavalli (2008) was applied. This phantom is a hermaphrodite model, which includes both male and female organs. Monte Carlo simulation was carried out for the emitted photons and electrons separately per decay of ${ }^{131} \mathrm{I}$ using this phantom. Afterward, the absorbed dose of the lung and the surrounding organs in $\mathrm{pGy} / \mathrm{MBq} . \mathrm{s}$ ( $S_{\text {lung to target }}$ ) using MCNPX2.4.0 (Briesmeister, 2000) with tally F6:e (the absorbed dose in the cell) was calculated. The relative errors associated with the Monte Carlo calculations were less than $3 \%$. The $S$ value, in turn, is defined as the mean absorbed dose to the target organ per unit of nuclear transition of the relevant radionuclide in the source region considered.

Studying the images collected from diagnosis and therapy imaging centers indicates that for those with a similar body size, the shape, volume and relative position of the internal organs can be significantly different. To ensure the existence of such differences, over 70 lung models extracted from chest computed tomography (CT) images collected from the Imam Hossein Hospital of Tehran, Imam Reza Hospital of Mashhad and Parsian Medical Imaging Center of Mashhad databases were investigated by Ebrahimi-Khankook et al. (2014, 2015).

Based on this information, considering fixed phantom organ dimensions while applying changes to the lung mass can be anatomically correct. After studying the difference in the lung mass in different people, it is vital to determine the range of the changes and its distribution. According to $\mathrm{Na}$ 's reports, which were published in 2009 and 2010, the mass (or the volume) normal distribution of the organs follows the Gaussian distribution with high reliability ( $\mathrm{Na}$ et al., 2009, 2010). The International Commission on Radiological Protection Publication 89 provided the standard anatomical and physiological information together with statistical data of some of the organs to protect patients from radiation (ICRP, 2002). In this journal, the mass of the lung and its standard deviation were reported to be $1246 \pm 322$ based on autopsies of 355 adult men.

To generate lungs with different masses, considering the average mass and its standard deviation, a FORTRAN program was applied to provide 98 random pieces of data with Gaussian distribution for the lung mass.

In the next stage, the 98 lungs were placed in the reference phantom, keeping the lung center of the mass fixed. Fixing the center of the mass causes the lung position to be the same in all of the phantoms.

In each of these 98 developed phantoms, the lung absorbed dose ( $\left.S_{\text {lung to lung }}\right)$ was calculated and is shown in Table 1 . The relative error due to Monte Carlo calculations for the lung absorbed dose was less than $0.1 \%$. Since the lung is the source and the target, the uncertainties associated with its calculation are smaller in comparison with the other organs which are far from the source.

\section{Results and discussion}

In nuclear medicine, photons from radiopharmaceuticals are used for diagnostic purposes. Since the injected activity for diagnosis is very small, even great differences in it are negligible. On the other hand, with respect to the high doses used for therapeutic purposes, considering even small differences in the dose is of vital importance. Therefore, accurate dosimetry calculations need to be made to diminish the risk of health effects associated with the administration of radiopharmaceuticals used for therapy (Han, 2005).

In this study, the lung was selected as the source of ${ }^{131} \mathrm{I}$, and the $S$ values for the lung and some other organs using the ORNL reference phantom were calculated. In addition, only the changes in the lung absorbed dose due to the changes in the lung mass were studied.

\subsection{The dose of the lung and other organs for the ORNL reference phantom}

The absorbed dose of the lung and the other target organs ( $\left.S_{\text {lung to target }}\right)$ for the total emitted energy of photons, electrons and their sum per decay of ${ }^{131} \mathrm{I}$ were calculated in pGy/Bq.s and are shown in Table 1 . As expected and according to the results, the lung receives the majority of the dose. It can be seen in Table 1 that since the majority of the electron energy is absorbed within the source, the lung absorbed dose resulting from electrons is higher than that from photons, while for the other organs, due to high escape of photons from the lungs, their absorbed doses resulting from photons are higher than those from electrons.

According to the low ranges of charged particles, the majority of the electron energy is absorbed within the source, only a marginal proportion of which is passed to the adjacent organs by Bremsstrahlung radiation. This marginal proportion is due to the Bremsstrahlung radiation taking place adjacent to heavy molecules and, on the other hand, the light and low-density tissue of the lung.

Considering the larger ranges of photons, other organs, in addition to the source, are going to have an uptake of the dose.

The organ absorbed dose is directly proportional to the organ absorbed energy of the photons and electrons per decay of a radionuclide. Considering that nearly all of the energy of the 
Table 1. The absorbed dose per the accumulated activity, $S$, for ${ }^{131} \mathrm{I}$ (pGy/Bq.s).

\begin{tabular}{cccc}
\hline \multirow{2}{*}{ Target organs } & \multicolumn{3}{c}{ Lung as source } \\
\cline { 2 - 4 } & Electrons per decay & Photons per decay & Electrons + photons \\
\hline Lung & $2.56 \times 10^{-2}$ & $3.64 \times 10^{-3}$ & $2.93 \times 10^{-2}$ \\
Heart wall & $5.82 \times 10^{-7}$ & $1.15 \times 10^{-3}$ & $1.15 \times 10^{-3}$ \\
Esophagus & $4.28 \times 10^{-7}$ & $1.02 \times 10^{-3}$ & $1.02 \times 10^{-3}$ \\
Breast & $1.85 \times 10^{-7}$ & $6.33 \times 10^{-4}$ & $6.33 \times 10^{-4}$ \\
Liver & $2.54 \times 10^{-7}$ & $4.09 \times 10^{-4}$ & $4.09 \times 10^{-4}$ \\
Spleen & $1.85 \times 10^{-7}$ & $3.27 \times 10^{-4}$ & $3.27 \times 10^{-4}$ \\
Stomach & $1.23 \times 10^{-7}$ & $2.57 \times 10^{-4}$ & $2.57 \times 10^{-4}$ \\
Skin & $3.85 \times 10^{-8}$ & $1.35 \times 10^{-4}$ & $1.35 \times 10^{-4}$ \\
\hline
\end{tabular}

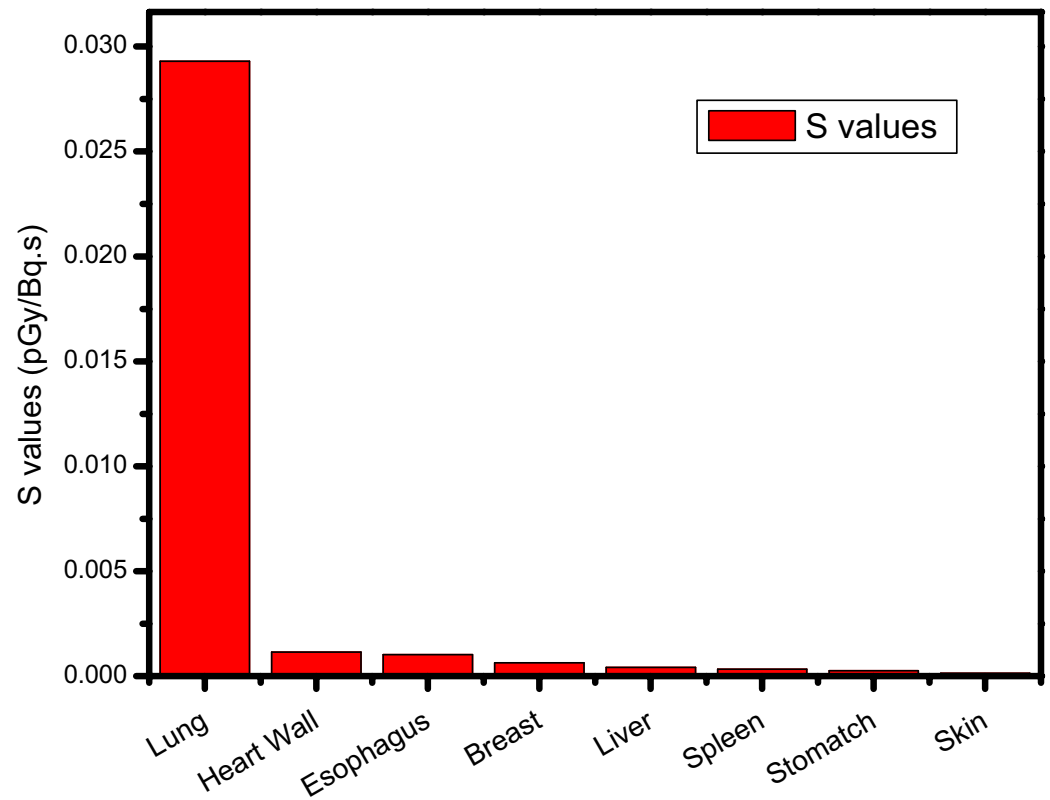

Figure 1. Comparison of $S$ values of the lung and other organs per decay of ${ }^{131} \mathrm{I}$.

Table 2. Comparison of the absorbed dose per the accumulated activity, $S$, for ${ }^{131} \mathrm{I}$ (pGy/Bq.s).

\begin{tabular}{cccc}
\hline Target organs & MIRDOSE3 & Snyder & This study \\
\hline Lung & $3.35 \times 10^{-5}$ & $3.38 \times 10^{-5}$ & $2.93 \times 10^{-5}$ \\
Heart wall & $1.18 \times 10^{-7}$ & - & $1.15 \times 10^{-7}$ \\
Esophagus & $5.6 \times 10^{-7}$ & - & $1.02 \times 10^{-6}$ \\
Breast & $5.44 \times 10^{-7}$ & $5.10 \times 10^{-7}$ & $4.09 \times 10^{-7}$ \\
Liver & $4.51 \times 10^{-7}$ & $4.65 \times 10^{-7}$ & $3.27 \times 10^{-7}$ \\
Spleen & $3.24 \times 10^{-7}$ & - & $2.57 \times 10^{-7}$ \\
Stomach & $1.35 \times 10^{-7}$ & $1.35 \times 10^{-7}$ & $1.35 \times 10^{-7}$ \\
\hline
\end{tabular}

electrons is absorbed within the source due to the low range of electrons, and most of the photon energy is also absorbed within the lung, its $S$ value is dramatically higher than the other organs' $S$ values (Figure 1).

A comparison of the results above with other studies is shown in Table 2 (Snyder et al., 1975; Clairand et al., 1999). The dose calculations were carried out using the Cristy and Eckerman mathematical phantom and DOSE3D Monte Carlo code in MIRDOSE3 software (Stabin, 1996). The deviation between the calculated dose to the lung in this study with those calculated by Snyder et al. and MIRDOSE3 software is about $13 \%$, which is associated with reasons such as the difference between incorporated phantoms and Monte Carlo codes. In other words, the applied lung masses in Snyder et al.'s study and MIRDOSE3 software were 1000 and 998 grams, respectively, while it was equal to 1246 grams in this study (ICRP, 2002). Considering that a specific organ mass differs from one person to another, one of the reasons for the deviation associated with phantoms is the variations in the organ mass. The coefficient of variation of the dose related to the lung mass has been calculated to be $18 \%$, which can fully explain the aforementioned 13\% discrepancy (Sajjadi et al., 2015). On the other hand, according to the ICRP Publication 116, the data sets generated from different Monte Carlo codes (such as MCNPX, 


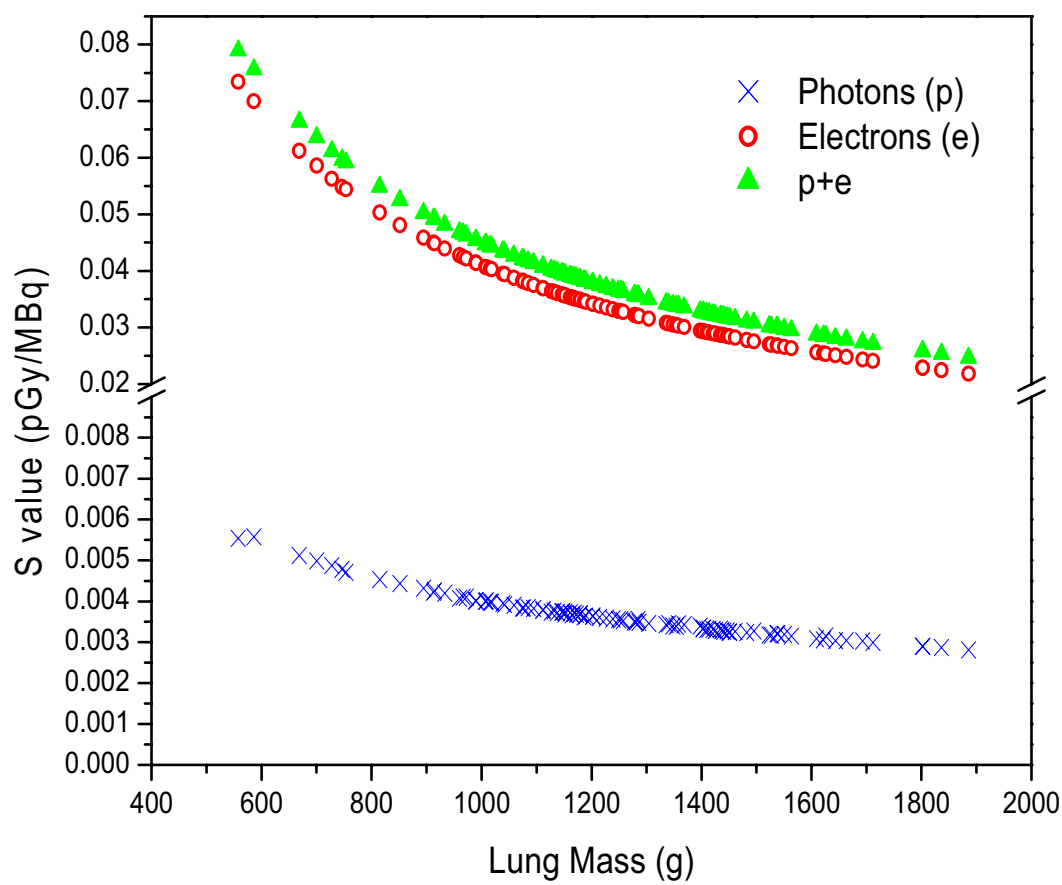

Figure 2. Changes in the lung absorbed dose versus its mass for photons, electrons and their sum per decay of ${ }^{131}$ I.

GEANT4 and DOSE3D) revealed that the variations among the results were relatively small. In the worst situation, the transport calculation method may result in up to $10 \%$ differences in organ absorbed doses (ICRP, 2010).

\subsection{Investigating changes in the lung absorbed dose with changes in its mass for ${ }^{131}$ I}

Regarding the high fraction of the dose deposited in the lung, variations in its absorbed dose are prominent, while the variations in the other organ doses, with regard to their low fraction of dose, are negligible. Hence, only the changes in the lung absorbed dose due to the changes in the lung mass were investigated.

Changes in the lung absorbed dose versus its mass are depicted in Figure 2 for photons, electrons and their sum per decay of ${ }^{131}$ I. Considering the slight differences between the two curves related to the absorbed dose for electrons and the absorbed dose for electrons + photons, it can be concluded that the electron proportion of the lung absorbed dose outweighs the photon proportion by a great deal since the energy deposited from photons is more dispersed around source regions relative to local deposition of the energy from electrons.

According to the inverse relationship between the dose and the mass, as the lung mass increases, its absorbed dose decreases. To study the rate of this reduction, the $S$ value as a function of the inverse of the mass $\left(\right.$ mass $\left.^{-1}\right)$ and as a function of the inverse of the surface (mass ${ }^{-2 / 3}$ ) in terms of the lung mass was plotted in Figure 3. To have an accurate comparison, the mass ${ }^{-1}$ and mass ${ }^{-2 / 3}$ figures were multiplied at a constant value to fit their start point. The changes in the lung surface are almost equal to mass ${ }^{2 / 3}$ (lungs can be considered as two halfellipsoids). In the mass distribution of the lung, the changes in the inverse of the mass and the surface of the largest lung to the smallest lung are almost $70 \%$ and $56 \%$, respectively. According to the aforementioned points, increasing the lung size results in a decrease in the ratio of the lung surface to the lung mass.

For electrons, the dose reduction of the largest lung relative to the smallest lung was calculated at about $70 \%$, which is almost proportional to the variations in the inverse of the lung mass (consistent with the agreement between the dose and the mass ${ }^{-1}$ curves in Figure 3a. Likewise, for photons and for the sum of photons and electrons, this reduction is about $49 \%$ and $69 \%$, respectively (Figs. $3 b$ and $3 c$ ). Therefore, for the electrons, our results are consistent with previous studies and representative of the fact that for $\beta$-emissions, $S$ values change with the inverse of the mass (Williams et al., 2002; Stabin et al., 2005):

$$
S_{2}=S_{1}\left(\frac{m_{1}}{m_{2}}\right)
$$

Zankl et al. showed that special absorbed fractions (SAFs) for large organs, such as the liver, are constant and agree with the inverse organ mass for electron energies up to approximately $1 \mathrm{MeV}$ (Zankl et al., 2012). It should be noted that they considered the organs consisting of soft tissue, whereas the lung density is one-third soft tissue. To investigate their assumption, $S$ values were calculated using Monte-Carlo code, considering the lung density to be equal to 0.26 (ICRP, 2002). It is noteworthy that there was a $26 \%$ discrepancy between our results and those calculated using Zankl et al.'s theory.

The dose (and $S$ value) for photons is inversely proportional to the mass with the power, ranging from $1 / 2$ to 1 at energies between 10 and $4000 \mathrm{keV}$ (i.e., 1/2 for $4000 \mathrm{keV}$ and 1 for $10 \mathrm{keV}$ ) (Eq. (2)) (Sajjadi et al., 2015), while according to Snyder's results and OLINDA/EXM software, it is 


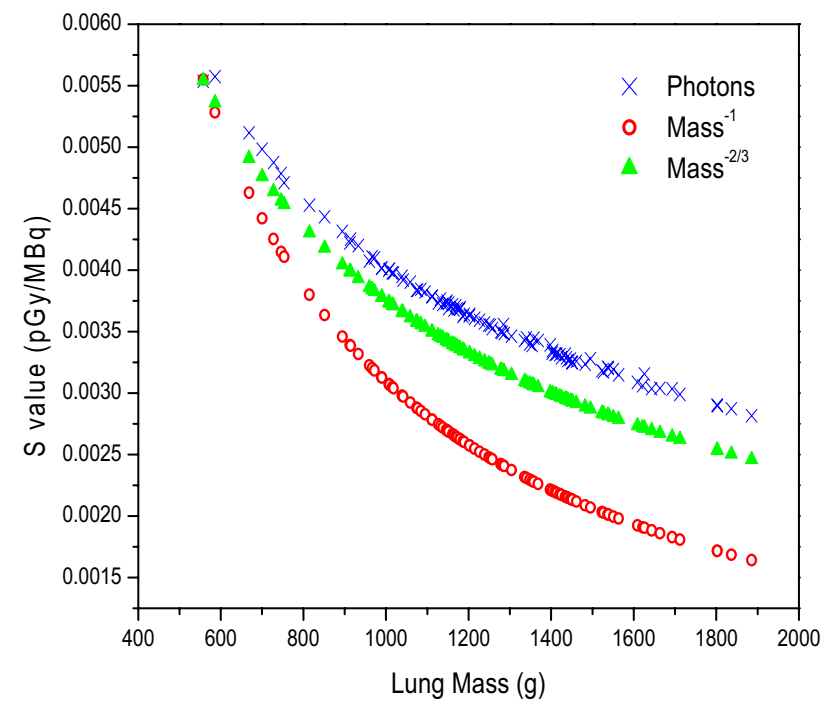

(a)

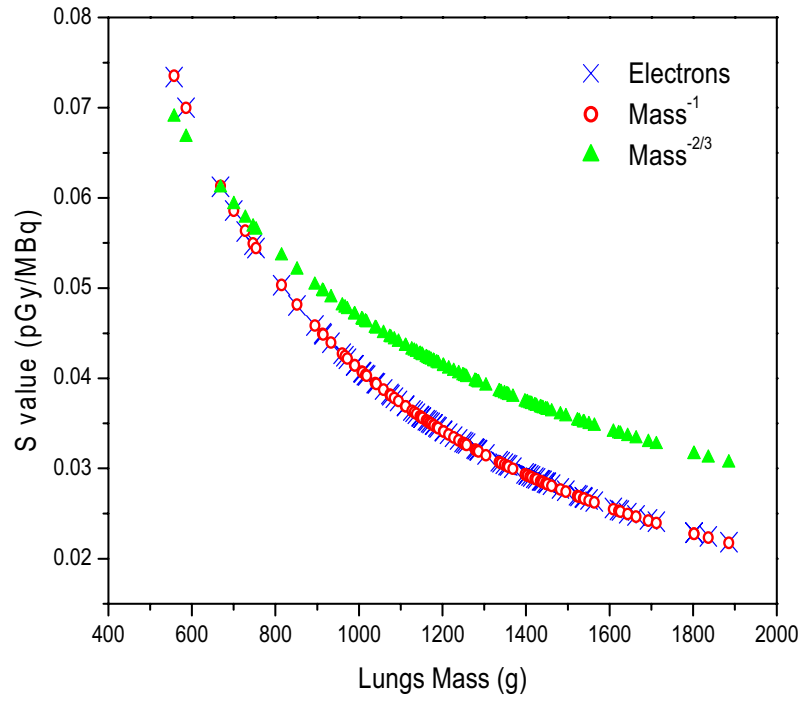

(b)

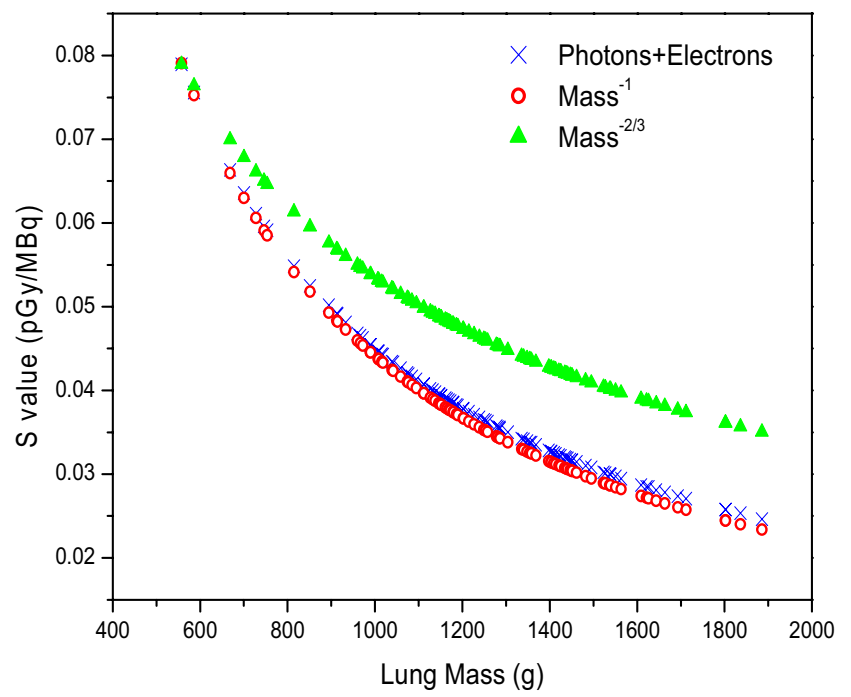

(c)

Figure 3. Comparison of the lung $S$ values, $S$ values as a function of the inverse of the mass $\left(\mathrm{mass}^{-1}\right)$ and as a function of the inverse of the surface $\left(\right.$ mass $^{-2 / 3}$ ) for (a) electrons, (b) photons and (c) electrons + photons per decay of ${ }^{131} \mathrm{I}$.

proportional to the mass ${ }^{-2 / 3}$ (Eq. (3)) (Snyder, 1970; Stabin et al. 2005; Hansson, 2012):

$$
\begin{aligned}
& S_{2}=S_{1}\left(\frac{m_{1}}{m_{2}}\right)^{x} ; \frac{1}{2} \leqslant x \leqslant 1 \\
& S_{2}=S_{1}\left(\frac{m_{1}}{m_{2}}\right)^{\frac{2}{3}} .
\end{aligned}
$$

Considering that the proportion of the electrons resulting from the decay of ${ }^{131} \mathrm{I}$ in the lung absorbed dose is much higher than that of the photons, similar to electrons, the changes in the dose for the sum of electrons and photons per decay are proportional to the inverse of the mass (Fig. 3c).

In addition, $S$ values for the right and left lung were calculated separately. The results were consistent with equations (1) and (2) for each of the lungs. Therefore, the cross-fire from one lung to the other does not break this law.

\section{Conclusion}

Regarding high doses due to the administration of radiopharmaceuticals for therapeutic purposes, this publication presents the effect of the lung mass on the lung absorbed dose, which was investigated per decay of ${ }^{131} \mathrm{I}$. Ninety-eight similar mathematical phantoms with different lung masses were implemented for precise dose calculations. The results show that the lung absorbed dose decreases with the increase in the lung mass, and mainly results from the absorbed energy of the electrons per decay of ${ }^{131} \mathrm{I}$. Considering that the other organ doses are much lower than the lung absorbed dose, the effect of variations in the lung mass is only significant for the lung absorbed dose: it is negligible for the other organs. It is highly recommended that further studies be carried out for other organ sources and their associated radionuclides used for radiotherapy. 


\section{References}

Briesmeister J.F. (2000) A general Monte Carlo N-particle transports code: version 4c. Report LA-13709-M, 1-427, Los Alamos National Laboratory, Los Alamos.

Brill A.B., Stabin M., Bouville A., Ron E. (2006) Normal organ radiation dosimetry and associated uncertainties in nuclear medicine, with emphasis on Iodine-131, Radiat. Res. 166 (1), 128-140.

Chen S., Yu L., Jiang C., Zhao Y., Sun D., Li S., Ye D. (2005) Pivotal Study of Iodine-131-Labeled Chimeric Tumor Necrosis Treatment radioimmunotherapy in Patients with advanced lung cancer, J. Clin. Oncol. 23 (7), 1538-1547.

Clairand I., Ricard M., Gouriou J., Di Paola M., Aubert B. (1999) DOSE3D: EGS4 Monte Carlo code-based software for internal radionuclide dosimetry, J. Nucl. Med. 40, 1517-1523.

Cristy M. (1985) Mathematical phantoms for use in reassessment of radiation doses to Japanese Atomic-Bomb survivors. Oak Ridge, TN: Oak Ridge National Laboratory. ORNL/TM-9487.

Ebrahimi-Khankook A., Miri-Hakimabad H., Rafat-Motavalli L. (2014) Studying the effect of the lung size variation on dosimetry and systematic parameters of FUM-IVNAA facility, $J$. Radioanal. Nucl. Chem. 303, 2263-2270.

Ebrahimi-Khankook A., Miri-Hakimabad H., Rafat-Motavalli L. (2015) A study of the effect of the lung shape on the lung absorbed dose in six standard photon and neutron exposure geometries, Radioprotection 50 (1), 65-72.

Halperin E.C., Brady L.W., Wazer D.E., Perez C.A. (2013) Perez $\mathcal{E}$ Brady's principles and practice of radiation oncology. Lippincott Williams \& Wilkins.

Han E.Y. (2005) Revised Series of Stylized Anthropometric Phantoms for Internal and External Radiation Dose Assessment, Ph.D Thesis, The University of Florida, pp. 16-36, 239-240.

Hansson E. (2012) The Internal Radiation Dosimetry of Diagnostic Radiopharmaceuticals across Different Asian Populations (Doctoral dissertation, Thesis, Sahlgrenska Academy, University of Gothenburg, Sweden.

ICRP Publication 23 (1975) Report of the Task Group on Reference Man.

ICRP Publication 89 (2002) Basic Anatomical and Physiological Data for Use in Radiological Protection: Reference Values. Pergamon, Oxford.

ICRP Publication 103 (2007) The 2007 Recommendations of the International Commission on Radiological Protection. Elsevier.

ICRP Publication 116 (2010) Conversion coefficients for radiological protection quantities for external radiation exposures. Oxford.

Kry S.F., Salehpour M., Followill D.S., Stovall M., Kuban D.A., White R.A., Rosen I.I. (2005) The calculated risk of fatal secondary malignancies from intensity-modulated radiation therapy, Int. J. Radiat. Oncol. Biol. Phys. 62 (4), 1195-1203.

Loevinger R., Japha E.M., Brownell G.L. (1956) Discrete radioisotope sources. In: Radiation Dosimetry (G.J. Hine, G.L. Brownell, Eds.), pp. 693-799. Academic Press, New York.
Miri-Hakimabad H., Rafat-Motavalli, L. (2008) Evaluation of specific absorbed fractions from internal photon sources in ORNL analytical adult Phantom, Radiat. Prot. Dosim. 128 (4), 427-431.

Mofrad F.B., Zoroofi R.A., Tehrani-Fard A.A., Akhlaghpoor S., Hori M., Chen Y.W., Sato Y. (2010) Statistical construction of a Japanese male liver phantom for internal radionuclide dosimetry, Radiat. Prot. Dosim. 141 (2), 140-148.

Na Y., Zhang J., Xu G., Hang B., Caracappa P.F. (2009) Nextgeneration deformable patient modeling for Monte Carlo assessment of organ doses, Med. Phys. 36, 2783.

Na Y.H., Zhang B., Zhang J.,Caracappa P.F., Xu X.G. (2010) Deformable adult human phantoms for radiation protection dosimetry: anthropometric data representing size distributions of adult worker populations and software algorithms, Phys. Med. Biol. 55 (13), 3789-3812.

Sajjadi Z., Miri-Hakimabad H., Rafat-Motavalli L., Mohammadi S. (2015) Evaluating the effects of statistical changes on internal dosimetry, Radioprotection 50 (3), 187-194.

Siegel R.L., Ma J., Zou Z., Jemal A. (2014) Cancer statistics, 2014, CA: a cancer journal for clinicians, 64 (1), 9-29.

Snyder W.S. (1970) Estimates of absorbed fraction of energy from photon sources in body organs (No. CONF-691212). Oak Ridge National Laboratory, Tennessee.

Snyder W.S., Ford M.R., Warner G.G., Watson S.B. (1975) "S" Absorbed dose per unit cumulated activity for selected radionuclides and organs. MIRD pamphlet no. 11. The Society of Nuclear Medicine, New York.

Stabin M.G. (1996) MIRDOSE: personal computer software for internal dose assessment in nuclear medicine, J. Nucl. Med. 37 (3), 538-546.

Stabin M.G. (2008) Uncertainties in internal dose calculations for radiopharmaceuticals, J. Nucl. Med. 49 (5), 853-860.

Stabin M.G., Watson E.E., Cristy M., Ryman J.C., Eckerman K.F., Davis J.L., Gehlen M.K. (1995) Mathematical models and specific absorbed fractions of photon energy in the nonpregnant adult female and at the end of each trimester of pregnancy (No. ORNL/TM-12907). Oak Ridge National Lab., TN (United States).

Stabin M.G., Sparks R.B., Crowe E. (2005) OLINDA/EXM: the second-generation personal computer software for internal dose assessment in nuclear medicine, J. Nucl. Med. 46 (6), 1023-1027.

Williams L.E., Liu A., Yamauchi D.M., Lopatin G., Raubitschek A.A., Wong J.Y. (2002) The two types of correction of absorbed dose estimates for internal emitters, Cancer 94 (S4), 1231-1234.

Yu L., Ju D.W., Chen W., Li T., Xu Z., Jiang C., Epstein, A.L. (2006) ${ }^{131}$ I-chTNT radioimmunotherapy of 43 patients with advanced lung cancer, Cancer Biotherapy Radiopharmaceuticals 21 (1), 514.

Zankl M., Schlattl H., Petoussi-Henss N., Hoeschen C. (2012) Electron specific absorbed fractions for the adult male and female ICRP/ICRU reference computational phantoms, Phys. Med. Biol. 57 (14), 4501.

Cite this article as: Z. Sajjadi, H. Miri-Hakimabad, L. Rafat-Motavalli. Studying the effects of the lung mass on the absorbed dose to the lung due to the administration of ${ }^{131}$ I for therapeutic purposes. Radioprotection 51(3), 187-192 (2016). 